\title{
Entanglement gain in measurements with unknown results
}

\author{
Margherita Zuppardo, ${ }^{1,2}$ Ray Ganardi, ${ }^{1}$ Marek Miller, ${ }^{1,3}$ Somshubhro Bandyopadhyay, ${ }^{4}$ and Tomasz Paterek ${ }^{1,5}$ \\ ${ }^{1}$ School of Physical and Mathematical Sciences, Nanyang Technological University, 637371 Singapore, Singapore \\ ${ }^{2}$ Science Institute, University of Iceland, IS-107 Reykjavik, Iceland \\ ${ }^{3}$ Instytut Fizyki Teoretycznej, Uniwersytet Wrocławski, Poland \\ ${ }^{4}$ Department of Physics and Center for Astroparticle Physics and Space Science, Bose Institute, EN80, Bidhannagar, Kolkata 700091, India \\ ${ }^{5}$ MajuLab, CNRS-UCA-SU-NUS-NTU International Joint Research Unit, UMI 3654 Singapore, Singapore
}

(Received 8 October 2018; published 10 April 2019)

\begin{abstract}
We characterize nonselective global projective measurements capable of increasing quantum entanglement between two particles. In particular, by choosing negativity to quantify entanglement, we show that entanglement of any pure nonmaximally entangled state can be improved in this way (but not of any mixed state) and we provide detailed analysis for two qubits. It is then shown that Markovian open system dynamics can only approximate such measurements, but this approximation converges exponentially fast as illustrated using the Araki-Żurek model. We conclude with numerical evidence that macroscopic bodies in a random pure state do not gain negativity in a random nonselective global measurement.
\end{abstract}

DOI: 10.1103/PhysRevA.99.042319

\section{INTRODUCTION}

Quantum entanglement is a prerequisite for many quantum information applications and tests of foundations of physics [1]. It is typically created in a sequence of unitary processes (quantum gates) applied to an initially disentangled system, whose environment typically inhibits the nonclassical correlations, see, e.g., [2-7]. The environment, however, does not always play the destructive role and another established route to entanglement generation is to engineer system-environment interactions in such a way that the decaying principal system reaches an entangled steady state, see, e.g., [8-12]. In this case, a manifold of initial system states evolve to the same entangled steady state.

Here we consider yet another possibility to generate or increase entanglement: via global projective measurements with unknown results. This may arise from the lack of ability to postselect a particular measurement result, or even in natural conditions, since any measurement can be approximated with suitable open system dynamics. Our approach is therefore related to engineering system-environment interactions but deviates from the decay processes because different initial states end up mostly in different final states having different amounts of entanglement. It is also impossible to prepare maximally entangled states through any nonselective measurement, but dissipation allows for such a possibility [10-12].

It turns out that measurements along maximally entangled bases are useless for any entanglement gain, modulo comments in Sec. II A. Nevertheless, entanglement can be increased as a result of nonselective measurement and, in fact, we show that entanglement of any pure nonmaximally entangled state can be enhanced in this way, and we provide a candidate optimal measurements for two qubits. Most of our results are derived by using negativity to quantify entanglement [13-16], the main reason for this choice being its easy computability for arbitrary dimensions.
Next we show an example of Markovian open system dynamics which rapidly approximates nonselective measurement on the principal system. In this way, we present another class of open system dynamics, additional to the decay processes, which increases entanglement in the system. Finally, we study negativity gain with random initial states and random measurements for growing dimensionality of the subsystems. We find that the probability of entanglement gain is negligible for higher dimensions. This could be seen as an element of quantum-to-classical transition showing that macroscopic objects, of high dimensionality, would have to be prepared in specific states and measured with specific nonselective measurements to entangle them.

\section{BLACK BOX PROBLEM}

We begin with the abstract formulation of the problem, described in Fig. 1. A bipartite quantum system in a general mixed state $\rho$ enters a black box where projective measurement $\left\{\Pi_{j}\right\}$ is conducted but its results remain unknown. That is, after the measurement, no single state $\Pi_{j}=\left|\psi_{j}\right\rangle\left\langle\psi_{j}\right|$ is selected for but rather we deal with a statistical mixture

$$
\rho^{\prime}=\sum_{j=0}^{D-1} \Pi_{j} \rho \Pi_{j},
$$

where $D=d_{1} d_{2}$ is the total dimension of tensor product space $\mathcal{H}_{1} \otimes \mathcal{H}_{2}$ of Hilbert spaces $\mathcal{H}_{1}$ and $\mathcal{H}_{2}$ of dimensions $d_{1}$ and $d_{2}$ describing each subsystem, respectively. Throughout the paper, we assume without loss of generality that $d_{1} \leqslant$ $d_{2}$. Our task is to study whether it is possible for quantum entanglement to increase as a result of the measurement, i.e., under what conditions the quantity

$$
\Delta E \equiv E\left(\rho^{\prime}\right)-E(\rho)
$$




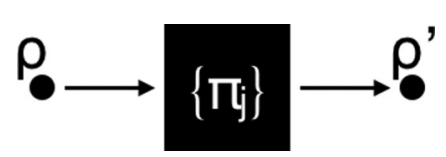

FIG. 1. Entanglement gain in nonselective global measurement as a black box problem. A state $\rho$ is input to a black box measuring device which conducts von Neumann measurement along projectors $\left\{\Pi_{j}\right\}$. Measurement results are unknown and therefore the state emerging from the box is $\rho^{\prime}=\sum_{j} \Pi_{j} \rho \Pi_{j}$. We study conditions under which entanglement in the state $\rho^{\prime}$ is higher than entanglement of the input state $\rho$.

is positive for a suitable entanglement measure $E$, chosen as negativity in this paper [13-16]. ${ }^{1}$

The following observation is an immediate consequence of the convexity of entanglement measure $E$ and Eq. (1). For any convex entanglement measure $E$

$$
E\left(\rho^{\prime}\right) \leqslant \max _{j} E\left(\Pi_{j}\right) .
$$

Note that inequality (3) holds also for entanglement monotones which are not convex, but nondecreasing functions of convex ones. A concrete example is the logarithmic negativity $L N(\rho)=\ln [2 N(\rho)-1]$, where we now recall a definition of the negativity $N(\rho)$ of the state $\rho$ as the sum of moduli of all negative eigenvalues of the partial transposition $\rho^{\Gamma}$ of $\rho$, and it is a convex function. However, there are entanglement measures to which this statement may not apply. Distillable entanglement, for instance, is conjured to be a nonconvex measure [17], and it is also unlikely to be a monotonic function of another convex measure.

We can rephrase this result as "there is no free lunch": to end up with an entangled state at the output there must be a supply of entanglement in the basis states along which one measures. Intuition would then suggest that it is desirable to choose the measurement basis states with as much entanglement as possible. It turns out that this is incorrect as the next section demonstrates.

\section{A. No gain with maximally entangled bases}

We now provide a proof that a measurement along any orthonormal basis composed of maximally entangled states of two qubits does not increase entanglement of any input state. The idea is to use the fact that any such basis can be transformed as a whole, by local unitary operations, to the Bell basis. $^{2}$ We then note that the nonselective measurement along the Bell basis can be implemented by local operations and classical communication (LOCC). Hence any measurement

\footnotetext{
${ }^{1}$ Note that if we allow general POVM measurements in place of projective von Neumann measurements the problem becomes trivial. There is a POVM that simply ignores the input to the box and always outputs maximally entangled pure state $\left|\psi^{+}\right\rangle$. For instance, take the POVM to be the collection of the following measurement operators: $M_{j}=\left|\psi^{+}\right\rangle\left\langle\psi_{j}\right|$.

${ }^{2}$ This is a folklore in the community, but since we were not able to locate a concrete reference we provide the complete proof as Lemma 1 in the Appendix.
}

along maximally entangled basis has LOCC realization and cannot increase entanglement. We will comment on higher dimensions after presenting the proof.

Theorem 1. Assume that $D=4$ and let every state $\left|\psi_{j}\right\rangle$ in the measurement basis be maximally entangled. Then for any input state $\rho$ and for any entanglement monotone with respect to LOCC, $E$ :

$$
E\left(\rho^{\prime}\right) \leqslant E(\rho)
$$

where $\rho^{\prime}=\sum_{j=0}^{3}\left|\psi_{j}\right\rangle\left\langle\psi_{j}|\rho| \psi_{j}\right\rangle\left\langle\psi_{j}\right|$.

Proof. By Lemma 1 (see Appendix), the basis $\left\{\left|\psi_{j}\right\rangle\right\}$ is locally unitarily equivalent to the Bell basis

$$
\begin{array}{ll}
\left|\psi_{0}\right\rangle=\frac{1}{\sqrt{2}}(|00\rangle+|11\rangle), & \left|\psi_{1}\right\rangle=\frac{1}{\sqrt{2}}(|01\rangle+|10\rangle), \\
\left|\psi_{2}\right\rangle=\frac{1}{\sqrt{2}}(|01\rangle-|10\rangle), \quad\left|\psi_{3}\right\rangle=\frac{1}{\sqrt{2}}(|00\rangle-|11\rangle) .
\end{array}
$$

It is a matter of straightforward calculation to verify that

$$
\begin{aligned}
& \left|\psi_{0}\right\rangle\left\langle\psi_{0}\right|=\frac{1}{4}\left(\mathbb{1} \otimes \mathbb{1}+\hat{\sigma}_{1} \otimes \hat{\sigma}_{1}-\hat{\sigma}_{2} \otimes \hat{\sigma}_{2}+\hat{\sigma}_{3} \otimes \hat{\sigma}_{3}\right), \\
& \left|\psi_{1}\right\rangle\left\langle\psi_{1}\right|=\frac{1}{4}\left(\mathbb{1} \otimes \mathbb{1}+\hat{\sigma}_{1} \otimes \hat{\sigma}_{1}+\hat{\sigma}_{2} \otimes \hat{\sigma}_{2}-\hat{\sigma}_{3} \otimes \hat{\sigma}_{3}\right), \\
& \left|\psi_{2}\right\rangle\left\langle\psi_{2}\right|=\frac{1}{4}\left(\mathbb{1} \otimes \mathbb{1}-\hat{\sigma}_{1} \otimes \hat{\sigma}_{1}-\hat{\sigma}_{2} \otimes \hat{\sigma}_{2}-\hat{\sigma}_{3} \otimes \hat{\sigma}_{3}\right), \\
& \left|\psi_{3}\right\rangle\left\langle\psi_{3}\right|=\frac{1}{4}\left(\mathbb{1} \otimes \mathbb{1}-\hat{\sigma}_{1} \otimes \hat{\sigma}_{1}+\hat{\sigma}_{2} \otimes \hat{\sigma}_{2}+\hat{\sigma}_{3} \otimes \hat{\sigma}_{3}\right),
\end{aligned}
$$

where, e.g., $\hat{\sigma}_{1}=|0\rangle\langle 1|+| 1\rangle\langle 0|$ stands for the $x$ Pauli operator. Using these equations one finds after direct calculation the postmeasurement state

$$
\begin{aligned}
\rho^{\prime}= & \frac{1}{4}\left(\rho+\hat{\sigma}_{1} \otimes \hat{\sigma}_{1} \rho \hat{\sigma}_{1} \otimes \hat{\sigma}_{1}\right. \\
& \left.+\hat{\sigma}_{2} \otimes \hat{\sigma}_{2} \rho \hat{\sigma}_{2} \otimes \hat{\sigma}_{2}+\hat{\sigma}_{3} \otimes \hat{\sigma}_{3} \rho \hat{\sigma}_{3} \otimes \hat{\sigma}_{3}\right) .
\end{aligned}
$$

The same postmeasurement state is obtained by the following LOCC procedure between the two observers, Alice and Bob. Alice selects at random number $j$ and informs Bob about the value of this classical random variable. Then Alice and Bob apply locally operation $\hat{\sigma}_{j} \otimes \hat{\sigma}_{j}$. Finally, they erase the record of the values of $j$. Therefore, for any entanglement monotone with respect to LOCC $E\left(\rho^{\prime}\right) \leqslant E(\rho)$.

Unfortunately extension of this method to higher dimensions is not straightforward. The proof of Lemma 1 in the Appendix does generalize and there is a similar LOCC procedure that returns the postmeasurement state which would be obtained by conducting nonselective measurement in the following straightforward generalization of the Bell basis [18]:

$$
\left|\psi_{j k}\right\rangle=\frac{1}{\sqrt{d}} \sum_{m=0}^{d-1} \omega_{d}^{m k}|m\rangle|m+j\rangle,
$$

where $j, k=0, \ldots, d-1, \omega_{d}=\exp (i 2 \pi / d)$, the sum in the last ket is modulo $d$, and for simplicity we assume that both systems have the same dimension $d$. However, it is not clear that any maximally entangled basis can be transformed by local unitaries to the basis (6).

\section{B. Pure input state}

Here we provide two theorems about the possibility of entanglement gain if pure states are presented at the input. From 
now on we will be quantifying entanglement by negativity, and our results are hence specific to this measure.

In Theorem 2 we construct an input state whose negativity increases in a given measurement. It turns out that all nontrivial von Neumann measurements, with individual basis vectors being neither product nor maximally entangled states in some subspace, increase entanglement of a suitable initial state. In Theorem 3 we construct a measurement which improves negativity of any pure input state which is not maximally entangled.

Theorem 2. Suppose that the measurement basis $\left\{\left|\psi_{j}\right\rangle\right\}$ is such that $\left|\psi_{0}\right\rangle$ has at least two nonzero Schmidt coefficients that are not equal. Then there exists a pure input state $\rho=$ $|\phi\rangle\langle\phi|$ for which negativity increases, i.e., $N\left(\rho^{\prime}\right)>N(\rho)$, where $\rho^{\prime}=\sum_{j=0}^{D-1}\left|\left\langle\psi_{j} \mid \phi\right\rangle\right|^{2}\left|\psi_{j}\right\rangle\left\langle\psi_{j}\right|$.

Proof. By assumption $\left|\psi_{0}\right\rangle=\sum_{i=0}^{d-1} \sqrt{p_{i}}|i i\rangle$, where $d \leqslant$ $d_{1}$, each $p_{i}>0$, and $p_{0} \neq p_{1}$. We construct the input state $|\phi\rangle$ as follows:

$$
|\phi\rangle \sim\left|\psi_{0}\right\rangle-\varepsilon|\Phi\rangle=\sum_{i=0}^{d-1}\left(\sqrt{p_{i}}-\varepsilon / \sqrt{d}\right)|i i\rangle,
$$

where $|\Phi\rangle=\frac{1}{\sqrt{d}} \sum_{i=0}^{d-1}|i i\rangle$ is the maximally entangled state defined using the Schmidt basis of $\left|\psi_{0}\right\rangle$. State $|\phi\rangle$ is normalized upon multiplication with constant $C^{-1}=\left(1+\varepsilon^{2}-\right.$ $2 \varepsilon \beta)^{-1 / 2}$, where $\beta \equiv\left\langle\psi_{0} \mid \Phi\right\rangle$. Our aim is to derive the range of $\varepsilon$ for which the postmeasurement state has more negativity than the input state $|\phi\rangle$. The postmeasurement state reads:

$$
\rho^{\prime}=(1-p)\left|\psi_{0}\right\rangle\left\langle\psi_{0}\right|+p \tilde{\rho},
$$

where $\tilde{\rho}$ lies in the orthogonal subspace to $\left|\psi_{0}\right\rangle$, and $p=1-$ $\left|\left\langle\psi_{0} \mid \phi\right\rangle\right|^{2}=\frac{\varepsilon^{2}}{C^{2}}\left(1-\beta^{2}\right)>0$ is the probability of obtaining the result different from zero. By the triangle inequality of the trace norm

$$
\begin{aligned}
\|\left|\psi_{0}\right\rangle\left\langle\left.\psi_{0}\right|^{\Gamma} \|_{1}\right. & \leqslant\left\|\rho^{\prime \Gamma}\right\|_{1}+p \| \tilde{\rho}^{\Gamma}-\left|\psi_{0}\right\rangle\left\langle\left.\psi_{0}\right|^{\Gamma} \|_{1}\right. \\
& \leqslant\left\|\rho^{\prime \Gamma}\right\|_{1}+2 d p .
\end{aligned}
$$

Since negativity of a generic state $\xi$ is given by $N(\xi)=$ $\left(\left\|\xi^{\Gamma}\right\|_{1}-1\right) / 2$ we find that

$$
N\left(\rho^{\prime}\right) \geqslant N\left(\left|\psi_{0}\right\rangle\left\langle\psi_{0}\right|\right)-p d .
$$

For the comparison with the initial state we calculate

$$
N(\rho)=\frac{1}{C^{2}}\left[N\left(\left|\psi_{0}\right\rangle\left\langle\psi_{0}\right|\right)+\varepsilon(\varepsilon-2 \beta) N(|\Phi\rangle\langle\Phi|)\right],
$$

where for simplicity we assumed that all the coefficients in (7) are positive, i.e.,

$$
\varepsilon<\sqrt{d} \min _{i} p_{i}
$$

We now calculate $N\left(\left|\psi_{0}\right\rangle\left\langle\psi_{0}\right|\right)$ from (11) and use it on the right-hand side of (10). The requirement of negativity gain, i.e., $N\left(\rho^{\prime}\right)>N(\rho)$, holds for positive $\varepsilon$ satisfying

$$
\varepsilon<\frac{2 \beta\left[N(|\Phi\rangle\langle\Phi|)-N\left(\left|\psi_{1}\right\rangle\left\langle\psi_{1}\right|\right)\right]}{d\left(1-\beta^{2}\right)+N(|\Phi\rangle\langle\Phi|)-N\left(\left|\psi_{1}\right\rangle\left\langle\psi_{1}\right|\right)} .
$$

We remind that $\varepsilon$ also has to satisfy (12).

A simple example shows that the condition in Theorem 2 is necessary. Consider two qutrits and suppose we do a

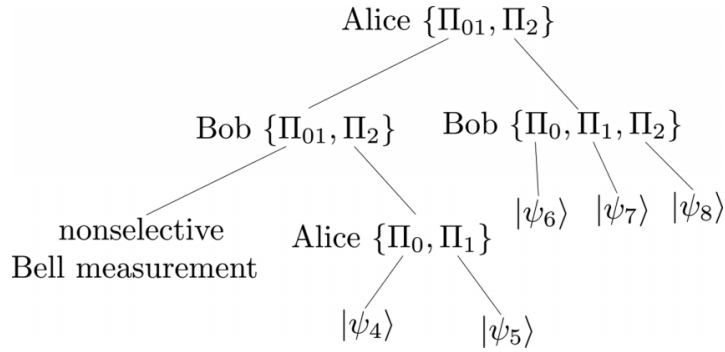

FIG. 2. Graphical depiction of an LOCC protocol to implement nonselective measurement in basis (14). Here $\Pi_{01}$ denotes $|0\rangle\langle 0|+$ $|1\rangle\langle 1|$, and $\Pi_{i}=|i\rangle\langle i|$. The LOCC procedure for the nonselective Bell measurement is described around Eq. (5).

nonselective measurement along the following basis:

$$
\begin{aligned}
& \left|\psi_{0}\right\rangle=\frac{1}{\sqrt{2}}(|00\rangle+|11\rangle), \quad\left|\psi_{1}\right\rangle=\frac{1}{\sqrt{2}}(|00\rangle-|11\rangle), \\
& \left|\psi_{2}\right\rangle=\frac{1}{\sqrt{2}}(|01\rangle+|10\rangle), \quad\left|\psi_{3}\right\rangle=\frac{1}{\sqrt{2}}(|01\rangle-|10\rangle), \\
& \left|\psi_{4}\right\rangle=|02\rangle, \quad\left|\psi_{5}\right\rangle=|12\rangle, \quad\left|\psi_{6}\right\rangle=|20\rangle, \\
& \left|\psi_{7}\right\rangle=|21\rangle, \quad\left|\psi_{8}\right\rangle=|22\rangle .
\end{aligned}
$$

Since the nonselective measurement along this basis is implementable by LOCC (see Fig. 2), it follows that the entanglement can never increase on average.

We have shown that for any nontrivial nonselective measurement, there exists a state whose negativity can be increased. Now we show that given any input pure state, which is not maximally entangled, one can find a projective nonselective measurement that increases the amount of entanglement of the input state.

Theorem 3. Suppose a pure initial state $|\phi\rangle$ is not maximally entangled. Then there exists a nonselective projective measurement $\left\{\Pi_{j}\right\}$ increasing negativity, i.e., $N\left(\sum_{j} \Pi_{j}|\phi\rangle\langle\phi| \Pi_{j}\right)>N(|\phi\rangle\langle\phi|)$.

Proof. Let us choose the measurement basis as follows:

$$
\begin{aligned}
& \left|\psi_{0}\right\rangle=\frac{1}{\sqrt{2+2\langle\phi \mid \Phi\rangle}}(|\phi\rangle+|\Phi\rangle), \\
& \left|\psi_{1}\right\rangle=\frac{1}{\sqrt{2-2\langle\phi \mid \Phi\rangle}}(|\phi\rangle-|\Phi\rangle),
\end{aligned}
$$

where $|\Phi\rangle=\frac{1}{\sqrt{d_{1}}} \sum_{i=0}^{d_{1}-1}|i i\rangle$ is the maximally entangled state having the same Schmidt basis as $|\phi\rangle$. One verifies that $\left|\psi_{0}\right\rangle$ and $\left|\psi_{1}\right\rangle$ are orthogonal, as they should be, and that $|\phi\rangle$ lies in the subspace they span, i.e., $|\phi\rangle=\alpha\left|\psi_{0}\right\rangle+\beta\left|\psi_{1}\right\rangle$. The other basis states are arbitrary as they do not contribute to the postmeasurement state

$$
\rho^{\prime}=|\alpha|^{2}\left|\psi_{0}\right\rangle\left\langle\left.\psi_{0}|+| \beta\right|^{2} \mid \psi_{1}\right\rangle\left\langle\psi_{1}\left|=\frac{1}{2}\right| \phi\right\rangle\left\langle\phi\left|+\frac{1}{2}\right| \Phi\right\rangle\langle\Phi|,
$$

where the second form of the state follows from (15). Since both $|\phi\rangle$ and $|\Phi\rangle$ have the same Schmidt basis, the final negativity is

$$
\begin{aligned}
N\left(\rho^{\prime}\right) & =\frac{1}{2}[N(|\phi\rangle\langle\phi|)+N(|\Phi\rangle\langle\Phi|)] \\
& =\frac{1}{2}\left[N(|\phi\rangle\langle\phi|)+\frac{1}{2}\left(d_{1}-1\right)\right]>N(|\phi\rangle\langle\phi|),
\end{aligned}
$$


where the value of negativity of the maximally entangled state was used.

\section{Mixed input state}

A similar statement to Theorem 3, but for all mixed input states, does not hold. To see this, recall that a state $\rho$ is termed absolutely separable [14] if $U \rho U^{\dagger}$ is separable for every unitary $U$ acting on $\mathcal{H}_{1} \otimes \mathcal{H}_{2}$. We will argue that the projective nonselective measurement can always be implemented as a mixed unitary channel acting on the input. Therefore, any absolutely separable input state leads to a separable postmeasurement state.

Proposition 1. The measurement map $\rho \rightarrow \sum_{j} \Pi_{j} \rho \Pi_{j}$ can be implemented as $\rho \rightarrow \sum_{j} p_{j} U_{j} \rho U_{j}^{\dagger}$, i.e., as a mixture of unitary operations.

Proof. By construction. Consider the unitary operator

$$
U \equiv \sum_{j=0}^{D-1} \omega_{d}^{j} \Pi_{j}
$$

The following probabilistic mixture mimics the nonselective measurement:

$$
\begin{aligned}
\frac{1}{D} \sum_{i=0}^{D-1} U^{i} \rho\left(U^{i}\right)^{\dagger} & =\frac{1}{D} \sum_{i, j, k=0}^{D-1} \omega_{d}^{i(j-k)} \Pi_{j} \rho \Pi_{k} \\
& =\sum_{j, k=0}^{D-1} \delta_{j k} \Pi_{j} \rho \Pi_{k} \\
& =\sum_{j=0}^{D-1} \Pi_{j} \rho \Pi_{j} .
\end{aligned}
$$

In the first line we used definition (18) and in the second line the following form of the Kronecker delta: $\sum_{i=0}^{D-1} \omega_{d}^{i(j-k)}=$ $D \delta_{j k}$.

The class of absolutely separable states is rather nontrivial. For example, it has been completely characterized for two qubits $[19,20]$. If $\lambda_{1} \geqslant \lambda_{2} \geqslant \lambda_{3} \geqslant \lambda_{4}$ are eigenvalues of $\rho$, then $\rho$ is absolutely separable, if and only if $\lambda_{3}+2 \sqrt{\lambda_{2} \lambda_{4}}-$ $\lambda_{1} \geqslant 0$. A concrete not absolutely separable mixed state, whose entanglement increases as a result of nonselective measurement, is seen as follows. Consider $\rho=\frac{1+2 \varepsilon}{3}|00\rangle\langle 00|+$ $\frac{1-\varepsilon}{3}|11\rangle\left\langle 11\left|+\frac{1-\varepsilon}{3}\right| 01\right\rangle\langle 01|$, for a small $\varepsilon>0$, and the basis as in (28) below, for $0<b<\frac{1}{\sqrt{2}}$ and $c=0$. The state $\rho$ is separable and $\varepsilon$-close to the set of absolutely separable states. It is easy to see, however, that the negativity of the postmeasurement state is strictly positive. It is an intriguing question for further research whether all the states not from this class can gain entanglement in a suitable nonselective measurement.

\section{Two qubits}

We now provide results on the optimal measurement, giving the highest negativity gain, for any pure input state. We begin with formulas valid for $d_{1}=d_{2}=d$ but soon restrict ourselves to $d=2$, i.e., two qubits. In the second case we derive the optimal choice of basis within a particular class of measurements. At the end of this section we provide numerical evidence that this measurement basis is actually the best possible choice in general.

Consider a pure input state $|\phi\rangle$, endowed with Schmidt decomposition

$$
|\phi\rangle=\sum_{i=0}^{d-1} \sqrt{p_{i}}|i i\rangle
$$

where $\sqrt{p_{i}}$ are Schmidt coefficients. We shall focus on the following specific choice of measurement basis. We assume that the first $d$ vectors are spanned by the Schmidt basis of the input state, i.e.,

$$
\left|\psi_{j}\right\rangle=\sum_{i=0}^{d-1} \alpha_{j i}|i i\rangle, \quad j=0, \ldots, d-1,
$$

and all the remaining vectors $\left|\psi_{j}\right\rangle$, with $j \geqslant d$, are orthogonal to $|\phi\rangle$. The state after the measurement reads

$$
\begin{aligned}
\rho^{\prime} & =\sum_{j=0}^{d-1}\left|\psi_{j}\right\rangle\left\langle\psi_{j} \mid \phi\right\rangle\left\langle\phi \mid \psi_{j}\right\rangle\left\langle\psi_{j}\right| \\
& =\sum_{j, i, i^{\prime}} \sum_{k, k^{\prime}} \sqrt{p_{i}} \alpha_{j i} \sqrt{p_{i^{\prime}}} \alpha_{j i^{\prime}}^{*} \alpha_{j k} \alpha_{j k^{\prime}}^{*}|k k\rangle\left\langle k^{\prime} k^{\prime}\right| .
\end{aligned}
$$

Negativity of this state admits the lower bound

$$
\begin{aligned}
N\left(\rho^{\prime}\right) & =\sum_{k<k^{\prime}}\left|\sum_{j, i, i^{\prime}} \sqrt{p_{i}} \alpha_{j i} \sqrt{p_{i^{\prime}}} \alpha_{j i^{\prime}}^{*} \alpha_{j k} \alpha_{j k^{\prime}}^{*}\right| \\
& \geqslant\left|\sum_{k<k^{\prime}} \sum_{j, i, i^{\prime}} \sqrt{p_{i}} \alpha_{j i} \sqrt{p_{i^{\prime}}} \alpha_{j i^{\prime}}^{*} \alpha_{j k} \alpha_{j k^{\prime}}^{*}\right| .
\end{aligned}
$$

It is therefore natural to define

$$
(\mathbf{m})_{i i^{\prime}}=\sum_{k<k^{\prime}} \sum_{j} \alpha_{j i} \alpha_{j i^{\prime}}^{*} \alpha_{j k} \alpha_{j k^{\prime}}^{*}, \quad(\mathbf{q})_{i}=\sqrt{p_{i}},
$$

where matrix $\mathbf{m}$ depends only on the measurement, and vector q depends only on the input state. Then

$$
N\left(\rho^{\prime}\right) \geqslant|\langle\mathbf{q}, \mathbf{m q}\rangle| .
$$

We now restrict ourselves to $d=2$. A pure two-qubit input state in its Schmidt basis reads

$$
|\psi\rangle=a|00\rangle+\sqrt{1-a^{2}}|11\rangle,
$$

where, without loss of generality, $a \in[0,1 / \sqrt{2}]$. Negativity of this input state is

$$
N_{i}=a \sqrt{1-a^{2}}
$$

We take the following basis of the projective nonselective measurement:

$$
\begin{aligned}
& \left|\psi_{1}\right\rangle=b|00\rangle+\sqrt{1-b^{2}}|11\rangle, \\
& \left|\psi_{2}\right\rangle=\sqrt{1-b^{2}}|00\rangle-b|11\rangle, \\
& \left|\psi_{3}\right\rangle=c|01\rangle+\sqrt{1-c^{2}}|10\rangle, \\
& \left|\psi_{4}\right\rangle=\sqrt{1-c^{2}}|01\rangle-c|10\rangle,
\end{aligned}
$$


where we note that the Schmidt basis of the input state is used to define every $\left|\psi_{j}\right\rangle$, but $\left|\psi_{3}\right\rangle$ and $\left|\psi_{4}\right\rangle$ do not contribute to the postmeasurement state. Using the previously introduced notation

$$
\begin{gathered}
\mathbf{q}=\left(a, \sqrt{1-a^{2}}\right), \\
\mathbf{m}=\left(\begin{array}{cc}
b & \sqrt{1-b^{2}} \\
\sqrt{1-b^{2}} & -b
\end{array}\right) .
\end{gathered}
$$

Hence, negativity of the relevant states of the measurement basis is fully characterized by parameter $b$ and we call the number $N_{b}=b \sqrt{1-b^{2}}$ the "negativity of the measurement." We choose $b \in[0,1 / \sqrt{2}]$. We observe that in the case of two qubits the outer sum in (23) contains just one term and the inequality (25) becomes an equality. The negativity of the final state $\rho^{\prime}$, expressed in terms of $N_{i}$ and $N_{b}$, is

$$
N_{f} \equiv N\left(\rho^{\prime}\right)=N_{b} \sqrt{1-4 N_{i}^{2}} \sqrt{1-4 N_{b}^{2}}+4 N_{i} N_{b}^{2} .
$$

The condition for entanglement gain, $N_{f}>N_{i}$, is realized for measurements and states that satisfy $N_{i}<N_{b}<\frac{1}{2}$, confirming what we already said about the lack of free lunch and the impossibility of entanglement enhancement in a measurement with maximally entangled basis states.

The best measurement for a given initial state (i.e., producing the largest final negativity) is obtained by solving $\frac{\partial N_{f}}{\partial N_{b}}=$ 0 for $N_{b}$. The corresponding negativity of the measurement reads

$$
N_{b}^{\max }=\frac{\sqrt{2 N_{i}+1}}{2 \sqrt{2}},
$$

and leads to entanglement gain decreasing linearly with the initial negativity

$$
N_{f}^{\max }-N_{i}=\frac{1}{4}-\frac{N_{i}}{2} .
$$

Therefore, the largest negativity gain is for initial product state measured along a basis having negativity $N_{b}^{\max }=1 / 2 \sqrt{2}$. This also shows again that any initial pure state has a strictly positive negativity gain in this process, unless it is maximally entangled.

Finally, we address the issue whether the results just presented are globally optimal. To derive them we assumed the measurement basis of the form (28). Although we were not able to provide a proof that this is indeed the optimal choice among all possible measurement bases, we conducted a numerical check which supports optimality. ${ }^{3}$ Figure 3 presents the data from a million of random trials, where each data point corresponds to a pair of random pure input state and random measurement. We plot negativity gain $N_{f}-N_{i}$ as a function of the negativity of the initial state $N_{i}$. We observe that the linear boundary derived in (33), for the measurement in (28) actually bounds from above all of the sampled cases.

\footnotetext{
${ }^{3}$ The codes, developed in PYTHON language, are available at in Ref. [21]
}

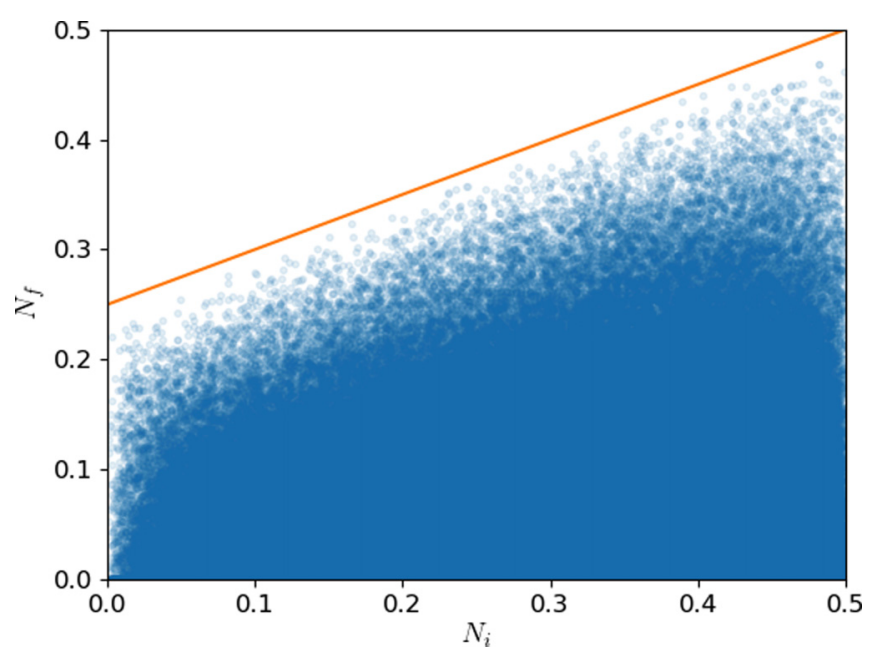

FIG. 3. Random sampling supports our claim that for the initial state (26) the measurement giving rise to highest entanglement gain is given by (28). Each point represents negativity gain for a random input state measured along random basis. Both states and measurements are sampled uniformly at random according to the Haar measures. One million trials are recorded. The upper boundary is given by (33) derived for the candidate optimal measurement, i.e., given by (28).

\section{PROJECTIVE MEASUREMENT IN OPEN SYSTEM DYNAMICS}

We have shown that nonselective global projective measurements can lead to entanglement gain in a bipartite system. Here we ask if such measurements could emerge as a result of open system dynamics. We demonstrate that a Markovian time evolution cannot lead to a perfect projective measurement in finite time. However, it rapidly converges to the projective measurement as illustrated using the Araki-Żurek model [22,23].

Suppose that $\mathcal{E}_{t}: \mathrm{M}_{n} \rightarrow \mathrm{M}_{n}$ is a Markovian evolution on the space of matrices of size $n$, which is norm-continuous and $\mathcal{E}_{0}=\mathbb{1}$. We require that this evolution reaches a projective map at least asymptotically, i.e., $\lim _{t \rightarrow \infty} \mathcal{E}_{t}=\Pi$, where $\Pi$ is a map with rank one projectors $\left\{\Pi_{j}\right\}$ as in our black box problem. It turns out that $\mathcal{E}_{t}$ cannot reach the projective map at any earlier time. The argument below shows that if this was the case the evolution would have to be a projector even for tiny evolution times which contradicts continuity, i.e., in this limit evolution map has to approach identity (nothing happens in very short times).

Theorem 4. $\mathcal{E}_{\tau} \neq \Pi$ for any finite time $\tau$.

Proof. By contradiction. Suppose there is finite $\tau$ such that $\mathcal{E}_{\tau}=\Pi$. Let $\Pi^{\prime}=\mathbb{1}-\Pi$, where $\mathbb{1}$ is the identity map, i.e., the action of $\Pi^{\prime}$ on a density matrix is to erase the diagonal elements in the basis defined by $\left\{\Pi_{j}\right\}$. We also define $\mathcal{E}_{t}^{\prime}=$ $\Pi^{\prime} \circ \mathcal{E}_{t}$ and therefore $\mathcal{E}_{\tau}^{\prime}=0$, where $\circ$ denotes composition of maps. Our aim is to show that $\mathcal{E}_{t}^{\prime}=0$ also for all $t<\tau$, i.e., $\mathcal{E}_{t}$ is a projector for all evolution times.

To this end we recall the definition of Markovianity $\mathcal{E}_{t+s}=$ $\mathcal{E}_{t} \circ \mathcal{E}_{s}=\mathcal{E}_{s} \circ \mathcal{E}_{t}$. Using it, one verifies a similar property for the primed maps:

$$
\mathcal{E}_{t+s}^{\prime}=\mathcal{E}_{t}^{\prime} \circ \mathcal{E}_{s}^{\prime}=\mathcal{E}_{s}^{\prime} \circ \mathcal{E}_{t}^{\prime} .
$$


Note that since $\mathcal{E}_{t}^{\prime}$ is a linear transformation, it can be represented as a $n^{2} \times n^{2}$ matrix. Let us now choose an integer $m$ and study matrix representation $A$ of the map $\mathcal{E}_{\tau / m}^{\prime}$ (we put no limit on how high $m$ could be). By our assumption and by property (34) we conclude that $A^{m}=0$. This implies that all the eigenvalues of matrix $A$ are zero, and therefore it has a simple Jordan normal form

$$
A=S\left(\begin{array}{cccc}
0 & 1 & & \\
& 0 & 1 & \\
& & \ddots & \\
& & & 0
\end{array}\right) S^{-1},
$$

with $1 s$ on suitable positions right above the diagonal and with $S$ being an invertible matrix. Since matrix $A$ has size $n^{2} \times n^{2}$, there exists an integer $\mu<n^{2}$ for which $A^{\mu}=0$ because multiplying $A$ with itself shifts the $1 s$ further from the diagonal. Since $\mu$ is independent of $m$ the map $\mathcal{E}_{\frac{\mu}{m} \tau}^{\prime}=$ 0 for any rational fraction of $\tau$. For any other $t$ we note using property (34) again that $\mathcal{E}_{t}^{\prime}=\mathcal{E}_{t-\frac{\mu}{m} \tau}^{\prime} \circ \mathcal{E}_{\frac{\mu}{m} \tau}^{\prime}=0$. We have therefore shown that $\mathcal{E}_{t}=\Pi$ for any ${ }^{m} 0<t^{m}<\tau$. Since the map $t \mapsto \mathcal{E}_{t}$ is continuous, it is required that $\mathcal{E}_{0}=\Pi$, which amounts to a contradiction.

To illustrate how open quantum dynamics can lead to a projective measurement, let us take a concrete example within the Araki-Żurek model [22,23]. Consider the system of two qubits coupled to the environment represented by a free particle moving on the real line. We wish to demonstrate entanglement gain for the initial state $\rho_{0}=|00\rangle\langle 00|$ and therefore choose the measurement basis

$$
\begin{aligned}
& \left|\psi_{0}\right\rangle=b|00\rangle+\sqrt{1-b^{2}}|11\rangle, \\
& \left|\psi_{1}\right\rangle=\sqrt{1-b^{2}}|00\rangle-b|11\rangle, \\
& \left|\psi_{2}\right\rangle=|01\rangle, \quad\left|\psi_{3}\right\rangle=|10\rangle,
\end{aligned}
$$

with $0<b<\frac{1}{\sqrt{2}}$. Recall, that the postmeasurement state reads $\rho^{\prime}=\sum_{j}\left\langle\psi_{j}|\rho| \psi_{j}\right\rangle\left|\psi_{j}\right\rangle\left\langle\psi_{j}\right|$.

Let us now define the total Hamiltonian for the evolution of the system and the environment. We put

$$
H=H_{S} \otimes \mathbb{1}_{E}+\mathbb{1}_{S} \otimes H_{E}+A \otimes B,
$$

where $H_{S}=\sigma_{z} \otimes \mathbb{1}-\mathbb{1} \otimes \sigma_{z}$ is the Hamiltonian for the system, $H_{E}=\frac{\hat{p}^{2}}{2 m}$ is the Hamiltonian of the environment and where $\hat{p}$ is the momentum operator of the particle with mass $m$. The interaction term is given by $A=\sum_{j} \lambda_{j}\left|\psi_{j}\right\rangle\left\langle\psi_{j}\right|$, with $\lambda_{j} \neq \lambda_{j^{\prime}}$ for $j \neq j^{\prime}$, together with $B=\hat{p}$. Observe that $\left[H_{S}, A\right]=0$ as well as $\left[H_{E}, B\right]=0$. The initial state of the environment is chosen to be $\omega_{E}=\left|\phi_{E}\right\rangle\left\langle\phi_{E}\right|$, where $\phi_{E}(x)=$ $\frac{1}{\sqrt{2 \pi}} \int \frac{e^{i x p}}{\sqrt{\pi\left(1+p^{2}\right)}} d p$ is the Cauchy distribution in momentum space here ensuring that the evolution is Markovian [24].

Starting from the uncorrelated initial state $\rho_{0} \otimes \omega_{E}$ the time evolution of the system alone is given by (7) in [24]:

$$
\rho_{t}=\sum_{n, m=0}^{3} e^{-\left|\lambda_{n}-\lambda_{m}\right| t} e^{i t\left(\gamma_{n}-\gamma_{m}\right)}\left\langle\psi_{n}\left|\rho_{0}\right| \psi_{m}\right\rangle\left|\psi_{n}\right\rangle\left\langle\psi_{m}\right|,
$$

where $\gamma_{0}=\gamma_{1}=0$ and $\gamma_{2}=-\gamma_{3}=2$. Thus

$$
\begin{aligned}
\rho_{t}= & b^{2}\left|\psi_{0}\right\rangle\left\langle\psi_{0}\left|+\left(1-b^{2}\right)\right| \psi_{1}\right\rangle\left\langle\psi_{1}\right| \\
& +e^{-|\mu| t} b \sqrt{1-b^{2}}\left(\left|\psi_{0}\right\rangle\left\langle\psi_{1}|+| \psi_{1}\right\rangle\left\langle\psi_{0}\right|\right),
\end{aligned}
$$

where $\mu=\lambda_{1}-\lambda_{2} \neq 0$. The negativity of $\rho_{t}$ is

$$
N\left(\rho_{t}\right)=b \sqrt{1-b^{2}}\left|2 b^{2}-1\right|\left(1-e^{-|\mu| t}\right) .
$$

It is easy to see that in the limit of $t \rightarrow \infty$ the system state approaches the postmeasurement state $\rho_{t} \rightarrow \rho^{\prime}$ in the trace norm. Note also that this convergence is exponentially fast, scaling as $\exp (-|\mu| t)$.

\section{RANDOM STATES AND RANDOM MEASUREMENTS}

Here we provide several results about entanglement gain with random measurements and random pure initial states on systems of different dimensions.

We found numerically that the probability of negativity gain is very small for $D=2 \times 2$ and $2 \times 3$ (respectively $\approx 1.6 \%$ and $\approx 0.17 \%$ ), whereas for higher $D$ we did not observe such cases at all, reinforcing the idea that such probability decreases with the dimension of Hilbert space. To understand this, we study the distribution of negativity in a random pure input state and compare it to the distribution of negativity in the postmeasurement state.

First we argue that the negativity distribution in the postmeasurement state is practically independent of the input state. Consider an input pure state $|\phi\rangle$ measured in a uniformly random basis. We denote by $P\left(N_{f} \mid \phi\right)$ the probability of observing an output state with negativity $N_{f}$. We have sampled from this distribution $10^{4}$ times for a given input state and numerically approximated it by dividing the range of negativity (note that it depends on $D$ ) into $10^{3}$ bins. In this way we discretize possible values of negativity justifying out notation $P\left(N_{f} \mid \phi\right)$ instead of probability density suitable for continuous variables. We then additionally sampled uniformly at random the input states, $10^{4}$ times, to approximate $P\left(N_{f}\right)$, probability distribution of negativity in the output state averaged over the input states. Table I shows that the two distributions are very similar as revealed by the estimated mean value of the statistical distance between them

$$
\mathcal{S}(\phi)=\sum_{N_{f}}\left|P\left(N_{f} \mid \phi\right)-P\left(N_{f}\right)\right| .
$$

We plot the distribution of negativity in the postmeasurement state in Fig. 4. For comparison we also present the negativity distribution in a randomly chosen input state. As clearly seen only for lower dimensions the two distributions have nonnegligible overlap. It is also clear that the chances of increasing negativity via a random measurement are negligible for dimensions of $3 \times 3$ and above.

TABLE I. Estimated mean of the statistical distance (41) obtained by sampling $10^{4}$ input states. It shows that the probability distribution of negativity in the postmeasurement state is practically independent of the input state.

\begin{tabular}{cc}
\hline \hline $\operatorname{dim}$ & $\langle\mathcal{S}\rangle$ \\
\hline $2 \times 2$ & 0.063 \\
$2 \times 3$ & 0.068 \\
$3 \times 3$ & 0.054 \\
$4 \times 4$ & 0.045 \\
\hline \hline
\end{tabular}




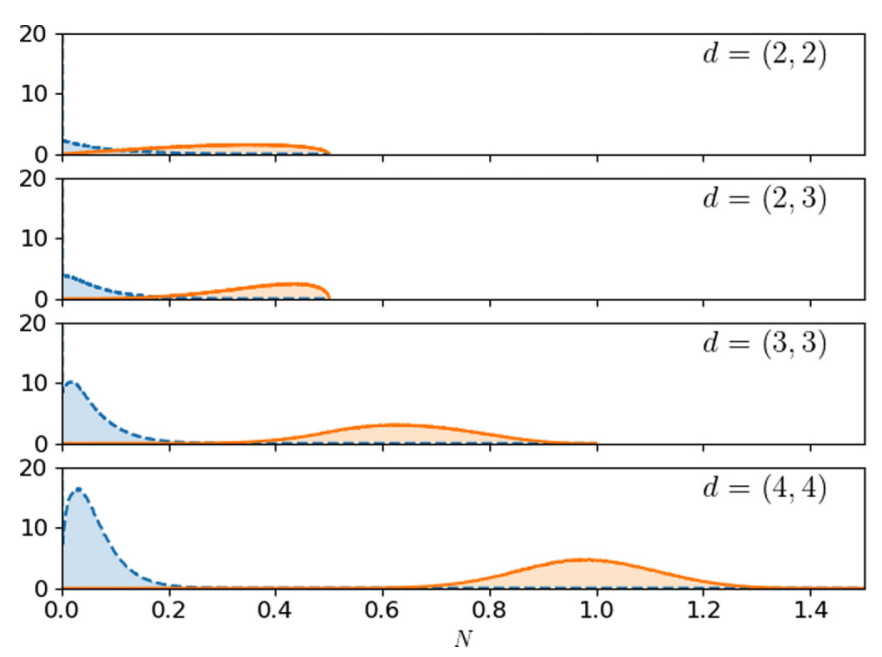

FIG. 4. Distribution of negativity between systems of different dimensions. Dashed lines enclosing blue color show the distributions for the postmeasurement state whereas solid lines enclosing orange color show the distribution for random pure input states.

We also notice that the statistics of negativity for the $2 \times 2$ and $2 \times 3$ cases are significantly different. In both such Hilbert spaces, the conditions of positivity under partial transposition and entanglement are equivalent to each other. Intuition may suggest a connection between the ability to increase negativity with unknown measurement results and the power of such quantity to detect entanglement. However, this connection appears to be weak, if at all present.

\section{CONCLUSION}

We propose the use of global nonselective projective measurements as means for entanglement creation or increase. Excellent approximations to such measurements may occur naturally in open system dynamics or they may also appear in engineered systems due to the lack of ability to postselect a particular measurement result. We show that entanglement of any pure nonmaximally entangled state can be improved in this way, but the final state can never be maximally entangled. In fact, measurements along bases with solely maximally entangled states provide no entanglement gain whatsoever (up to comments in Sec. II A). We derive candidate optimal measurements, giving maximal negativity gain, for any pure input state of two qubits. Numerical checks support the optimality. It turns out that it is best to start with a product state and measure it along the basis with states having a moderate negativity of $1 / 2 \sqrt{2}$. We hope these results on entanglement gain will find concrete realizations leading to new ways of generating this important physical resource.

\section{ACKNOWLEDGMENTS}

We thank Michał Horodecki, Kavan Modi, Aby Philip, and Somasundaram Sankaranarayanan for discussions. S.B. thanks Anindita Banerjee and Saronath Halder for helpful discussions. This work is supported by Singapore Ministry of Education Academic Research Fund Tier 2 Project No. MOE2015-T2-2-034. S.B. is supported, in part, by SERB
Project No. EMR/2015/002373. M.Z. is supported by the Icelandic Research Fund, Grant No. 163082-051. Some of the computations were performed on resources provided by the Icelandic High Performance Computing Centre at the University of Iceland.

\section{APPENDIX}

Lemma 1. Let $d_{1}=d_{2}=2$. Assume that every state $\left|\psi_{j}\right\rangle$ of an orthonormal basis is maximally entangled. Then the basis is locally unitarily equivalent to the Bell basis.

Proof. Let $\left\{\left|a_{k} b_{l}\right\rangle\right\}$ be a product-vector basis, for which $\left|\psi_{0}\right\rangle=\frac{1}{\sqrt{2}}(|00\rangle+|11\rangle)$. There always exists such a basis due to Schmidt decomposition. We expand the rest of the states $\left|\psi_{j}\right\rangle$ in the same basis

$$
\left|\psi_{j}\right\rangle=\sum_{k, l=0}^{1} \alpha_{k l}^{(j)}\left|a_{k} b_{l}\right\rangle,
$$

and consider $2 \times 2$ matrices $\alpha^{(j)}$ with entries given by the coefficients of the state $\left|\psi_{j}\right\rangle$. They have the following properties stemming from maximal entanglement and orthonormality of these states:

$$
\begin{aligned}
\text { max. entanglement } & \Longleftrightarrow \alpha^{(j)} \text { unitary, } \\
\left\langle\psi_{j} \mid \psi_{j^{\prime}}\right\rangle=\delta_{j j^{\prime}} & \Longleftrightarrow \operatorname{Tr}\left[\alpha^{(j)}\left(\alpha^{\left(j^{\prime}\right)}\right)^{\dagger}\right]=\delta_{j j^{\prime}} .
\end{aligned}
$$

Note that $\operatorname{Tr}\left(\alpha^{(j)}\right)=0$ for $j=1,2,3$. Any traceless $2 \times 2$ unitary matrix is proportional to a Hermitian one, i.e., each $\alpha^{(j)}=e^{i \eta_{j}} A^{(j)}$, where $A^{(j)}$ is Hermitian. Since multiplying matrices $\alpha^{(j)}$ by a phase factor $e^{i \eta_{j}}$ does not change the postmeasurement state $\rho^{\prime}$, we can assume without loss of generality that each $\alpha^{(j)}$ itself is Hermitian, for $j=1,2,3$. The set $\left\{\frac{1}{\sqrt{2}} \mathbb{1}, \alpha^{(1)}, \alpha^{(2)}, \alpha^{(3)}\right\}$ is therefore an orthonormal basis in the four-dimensional real Hilbert space of Hermitian $2 \times 2$ matrices with the Hilbert-Schmidt inner product. Since the same space is spanned by the identity and the Pauli matrices there is an isometry $R$ such that

$$
\mathbb{1}=R \mathbb{1}, \quad \alpha^{(j)}=R\left(\frac{1}{\sqrt{2}} \sigma_{j}\right) \quad \text { for } j=1,2,3,
$$

where $\sigma_{j}$ is the $j$ th Pauli matrix. The matrix representation of the mapping $R$ reads

$$
R=\left(\begin{array}{cc}
1 & 0 \\
0 & R_{0}
\end{array}\right)
$$

where $R_{0} \in \mathrm{O}(3)$ is an orthogonal matrix. We can always assume that $R_{0} \in \mathrm{SO}(3)$. Indeed, if $\operatorname{det} R_{0}=-1$, we would take one of the measurement basis vectors, say $\left|\psi_{1}\right\rangle$, with the opposite sign, which amounts to a global phase change. That would change the sign of $\alpha^{(1)}$ and consequently the sign of $\operatorname{det} R_{0}$, but the postmeasurement state $\rho^{\prime}$ would stay the same.

Using the homomorphism between the groups $\mathrm{SU}(2)$ and $\mathrm{SO}(3)$ we infer that there exists a unitary matrix $U \in \mathrm{SU}(2)$, such that $\alpha^{(j)}=\frac{1}{\sqrt{2}} R_{0} \sigma_{j}=\frac{1}{\sqrt{2}} U \sigma_{j} U^{\dagger}$, for $j=1,2,3$, and therefore

$$
\left|\psi_{j}\right\rangle=\frac{1}{\sqrt{2}} \sum_{k, l=0}^{1}\left(U \sigma_{j} U^{\dagger}\right)_{k l}\left|a_{k} b_{l}\right\rangle .
$$


Note that last equation holds for all $j$ because $\alpha^{(0)}=\mathbb{1}$. By opening up matrix multiplication $U \sigma_{j} U^{\dagger}$ and defining new local bases

$$
|m\rangle \equiv \sum_{k} U_{k m}\left|a_{k}\right\rangle, \quad|n\rangle \equiv \sum_{l} U_{l n}^{*}\left|b_{l}\right\rangle,
$$

the initial maximally entangled basis is brought to the form

$$
\left|\psi_{j}\right\rangle=\frac{1}{\sqrt{2}} \sum_{m, n=0}^{1}\left(\sigma_{j}\right)_{m n}|m n\rangle .
$$

This is, up to the irrelevant global phase of $\left|\psi_{2}\right\rangle$, the standard Bell basis.
[1] R. Horodecki, P. Horodecki, M. Horodecki, and K. Horodecki, Rev. Mod. Phys. 81, 865 (2009).

[2] B. Julsgaard, A. Kozhekin, and E. S. Polzik, Nature 413, 400 (2001).

[3] M. Steffen, M. Ansmann, R. C. Bialczak, N. Katz, E. Lucero, R. McDermott, M. Neeley, E. M. Weig, A. N. Cleland, and J. M. Martinis, Science 313, 1423 (2006).

[4] R. Blatt and D. Wineland, Nature 453, 1008 (2008).

[5] T. Palomaki, J. Teufel, R. Simmonds, and K. Lehnert, Science 342, 710 (2013).

[6] M. Jabir and G. Samanta, Sci. Rep. 7, 12613 (2017).

[7] R. Riedinger, A. Wallucks, I. Marinković, C. Löschnauer, M. Aspelmeyer, S. Hong, and S. Gröblacher, Nature 556, 473 (2018).

[8] H. Krauter, C. A. Muschik, K. Jensen, W. Wasilewski, J. M. Petersen, J. I. Cirac, and E. S. Polzik, Phys. Rev. Lett. 107, 080503 (2011).

[9] F. Verstraete, M. M. Wolf, and J. I. Cirac, Nat. Phys. 5, 633 (2009).

[10] Y. Lin, J. Gaebler, F. Reiter, T. R. Tan, R. Bowler, A. Sørensen, D. Leibfried, and D. J. Wineland, Nature 504, 415 (2013).

[11] S. Shankar, M. Hatridge, Z. Leghtas, K. Sliwa, A. Narla,
U. Vool, S. M. Girvin, L. Frunzio, M. Mirrahimi, and M. H. Devoret, Nature 504, 419 (2013).

[12] F. Reiter, D. Reeb, and A. S. Sørensen, Phys. Rev. Lett. 117, 040501 (2016).

[13] G. Vidal and R. F. Werner, Phys. Rev. A 65, 032314 (2002).

[14] K. Życzkowski, P. Horodecki, A. Sanpera, and M. Lewenstein, Phys. Rev. A 58, 883 (1998).

[15] J. Lee and M. S. Kim, Phys. Rev. Lett. 84, 4236 (2000).

[16] J. Lee, M. Kim, Y. Park, and S. Lee, J. Mod. Opt. 47, 2151 (2000).

[17] P. W. Shor, J. A. Smolin, and B. M. Terhal, Phys. Rev. Lett. 86, 2681 (2001).

[18] C. H. Bennett, G. Brassard, C. Crépeau, R. Jozsa, A. Peres, and W. K. Wootters, Phys. Rev. Lett. 70, 1895 (1993).

[19] S. Ishizaka and T. Hiroshima, Phys. Rev. A 62, 022310 (2000).

[20] F. Verstraete, K. Audenaert, and B. De Moor, Phys. Rev. A 64, 012316 (2001).

[21] http://github.com/rganardi/random-measurement.

[22] H. Araki, Prog. Theor. Phys. 64, 719 (1980).

[23] W. H. Zurek, Phys. Rev. D 26, 1862 (1982).

[24] P. Blanchard and R. Olkiewicz, Rev. Math. Phys. 15, 217 (2003). 